\title{
ATROPHY OF THE THALAMUS IN A CASE OF AC- QUIRED HEMIPLEGIA ASSOCIATED WITH DIF- FUSE PORENCEPHALY AND SCLEROSIS OF THE LEFT CEREBRAL HEMISPHERE
}

BY

\author{
W. E. LE GROS CLARK AND D. S. RUSSELL *
}

From the Department of Anatomy, Oxford, and the Bernhard Baron Institute of Pathology, the London Hospital

(ReCEIVED 1st December, 1939)

THE following case is reported both on account of its unusual pathological features and the opportunity it affords of studying thalamic atrophy in an example of what is for all practical purposes the equivalent of an experimental hemidecortication in a human subject. In recent years a considerable amount of experimental work has been completed on the study of thalamo-cortical connections in lower mammals. This work has demonstrated that the projection of different thalamic nuclei on to different areas of the cortex is more precise and also more extensive than had been generally supposed. In experimental animals, the degree to which thalamic nuclei are functionally dependencies of the cortex has been assessed in some cases by the study of cell degeneration in the thalamus following complete, or almost complete, hemidecortication.

Since in the present case the pathological condition has led to a destruction of the cortex while the basal ganglia have been left intact, it also permits of a differentiation between thalamo-cortical connections, and possible thalamostriate connections which may exist (and which have been described) in respect of some of the nuclear elements of the thalamus.

\section{Clinical History}

M. B., a girl aged thirteen years, was first admitted when eight years old to the wards of the London Hospital on 8th August, 1933, under the care of Dr. Donald Hunter, to whom we are indebted for the following clinical notes.

She had complained of occasional headaches during the preceding three weeks. On the day of admission a sudden attack of vomiting was followed by convulsions affecting the right side of the body, and unconsciousness. She had previously been healthy. There was no history of epilepsy or of other neurological diseases in the family. One sister, aged four and a half years, was alive and well.

On Examination.- She was obviously ill and in a state of cerebral irritability.

* Working for the Medical Research Council. 
Temperature $105 \cdot 5^{\circ} \mathrm{F}$. Pulse 150 . The fundi appeared normal. There was conjugate deviation of both eyes to the right and nystagmus. The reactions of the pupils were normal. There were clonic convulsions of the right hand and right face. The knee and ankle jerks were absent and an extensor plantar response was obtained on the right side. No abnormal physical signs were found in the chest or abdomen.

On 9th August a right hemiplegia and right facial paralysis developed. Some evidence of infection of the post-nasal region and of the left middle ear was found, but needling of the left temporal lobe through a trephine hole yielded cerebro-spinal fluid and thus it was concluded that no abscess was present. On 10th August there was slight neck rigidity and Kernig's sign was positive on the right side. The left arm and leg moved in response to pin-prick.

A later note states that a flaccid paralysis of the right arm and leg with increased tendon reflexes persisted. The general condition improved and by 28th August "she was able to recite nursery rhymes." (This may be taken to imply that there had been some impairment of speech at an earlier stage.) On 8th September it is noted that she appeared well and happy, talked freely, and was normal mentally. On 16th September a slight degree of apraxia was recorded and a greater degree of spasticity of the right limbs. She was able to kick with the right lower limb and to move it fairly freely. Movements of the right upper limb were beginning and there was less weakness of the right face. Both plantar reflexes were extensor, but no other abnormal signs were found in the left leg. On 23 rd October the changes in the nervous system had become stationary and she was discharged to a convalescent home wearing a surgical boot.

The following were the findings of the laboratory investigations.

Cerebro-spinal Fluid.-9th August (a) by lumbar puncture : clear fluid at $180 \mathrm{~mm}$. pressure ; 0.035 per cent. of protein ; 0.73 per cent. of chlorides ; 53 cells per $\mathrm{cmm}$., mostly lymphocytes. Cultures sterile. Wassermann reaction negative.

(b) From lateral ventricle : 0.035 per cent. of protein : no excess of white cells.

10th August. Lumbar puncture : protein, 0.045 per cent. ; 96 cells per cmm., 60 per cent. lymphocytes and 40 per cent. neutrophil leucocytes.

Urine.-9th August. Albumin, a cloud; a few red blood-corpuscles, occasional leucocytes and epithelial cells in deposit.

On 18th May, 1936, the child was re-admitted on account of attacks of unconsciousness lasting about 30 seconds. These had started soon after her discharge from hospital, occurring at first about twice a week and then becoming more frequent. There was no headache. On examination it was found that intelligence and speech were normal. There was no pyrexia. A right homonymous hemianopia was recorded, but the notes do not contain charts of the visual fields. Weakness of the right face was apparent during voluntary movements. The tongue was protruded slightly to the right. The right upper limb showed diminished power, hypertonia, and increased tendon reflexes without wasting. The abdominal reflexes were absent on the right, present on the left. The right lower limb showed $2.5 \mathrm{~cm}$. of shortening with contracture of the Achilles tendon, spasticity, and diminished power. The deep reflexes were exaggerated ; there were patellar and ankle clonus and an extensor plantar response on the right. No sensory disturbance was detected.

Several fits were observed while she was in hospital. These came on suddenly without an aura. In them she cried and fell to the left, remaining unconscious for about 30 seconds. Afterwards she was dazed for a short time, and this was followed by involuntary jerky movements of the right arm and leg. There was no incontinence of urine nor biting of the tongue. The attacks continued and, when on 15th July, 1938, she was again re-admitted, she was in status epilepticus. Two weeks before this she had had an attack of diarrhœa and vomiting. Eight days before admission convulsions had become almost continuous. Examination on this admission revealed the following 
signs : There was twitching of the eyelids, mouth, right hand and leg, followed by tonic and clonic contractions. Sedative drugs were ineffective. Unconsciousness persisted, and after further fits she died on 17th July, 1938.

The brain was injected with formaldehyde through the right carotid artery on the day of death. The necropsy was performed on the following day, and we are indebted to Dr. J. R. Gilmour for the following summary.

\section{Summary of Necropsy}

Status Epilepticus. Atrophy and Sclerosis of Left Cerebrum.-Severe parenchymatous degeneration of myocardium. Slight general atheroma. Broncho-pneumonic consolidation and almost complete collapse of lower lobe of right lung. Hæmorrhage between posterior common ligament and sides of lower thoracic and lumbar vertebræ. Moderate fatty degeneration of liver. Slight albuminous degeneration of kidneys. Middle ears and accessory nasal sinuses normal. Slight generalized pigmentation of skin. Healed trephine wound in skull. Right lower limb $2 \mathrm{~cm}$. shorter than left; right upper limb $1.5 \mathrm{~cm}$. shorter than left. A well-nourished, otherwise well-developed, girl. Height $160 \mathrm{~cm}$. Weight $48 \cdot 65 \mathrm{~kg}$.

\section{Pathology of Central Nervous System}

Macroscopic Examination.-The left cerebral hemisphere was greatly atrophied (Fig. 1), all areas being affected except the anterior two-thirds of the cingulate gyrus, the uncus and adjacent medial part of the hippocampus, including the dentate gyrus, a narrow strip of the inferior temporal convolution, the lips of the calcarine fissure, and the terminal $1.5 \mathrm{~cm}$. of the occipital pole. Over these parts of the hemisphere the leptomeninges were thin and of normal transparency; elsewhere they appeared cloudy and greyish-white, and in places were thrown into irregular folds (Fig. 1). The atrophied convolutions felt soft and spongy. The right cerebral hemisphere appeared normal. The right cerebellar hemisphere was slightly smaller than the left due to a generalized but unequal atrophy of the folia.

Both internal carotid arteries were of equal size and of normal translucency ; the main cerebral arteries were patent and of normal appearance on both sides.

On section the cortex of the left cerebrum, except in the unaffected areas mentioned, was found to be replaced by a thin layer of soft, grey, spongy tissue composed of delicate trabeculæ enclosing empty spaces mostly less than $0 \cdot 1 \mathrm{~cm}$. diameter but occasionally measuring up to $0 \cdot 5 \mathrm{~cm}$. diameter (Figs. 2, 3). Deep to this porous layer and throughout the hemisphere the white matter was grey and of a curious rubbery consistency suggestive of wash-leather. In the left paracentral lobule the cortex was not porous, but its outline was blurred and the white matter tough and rubbery as elsewhere. In addition there were occasional areas in the more grossly affected parts of the convexity where the cortex was homogeneous and yellowish-grey; such foci tended to occupy the depths of the sulci. 


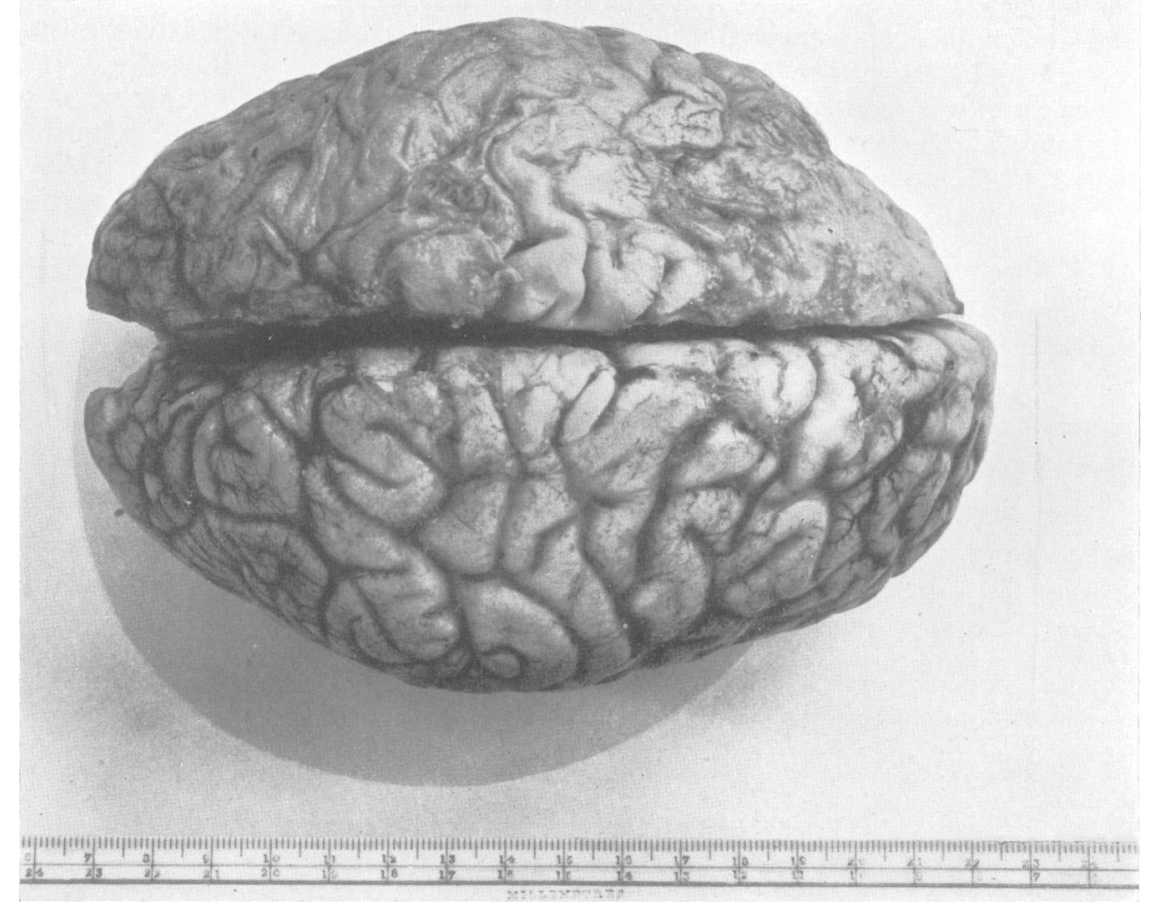

Fig. 1.-Dorsal view of brain showing atrophy of left cerebral hemisphere. Note thickening and corrugation of leptomeninges.

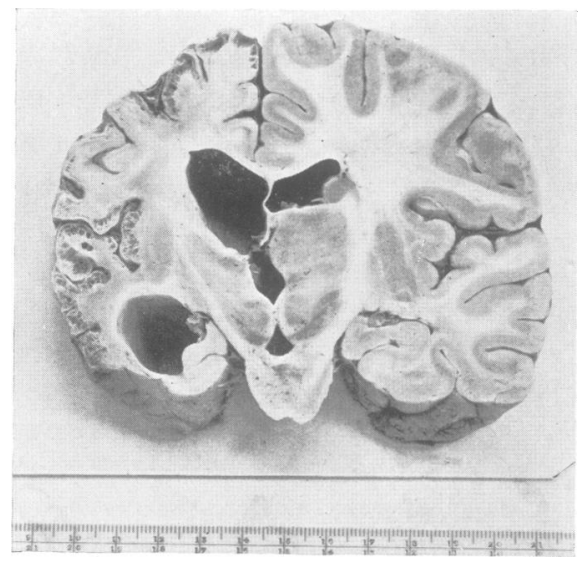

Fig. 2.- Section of the brain at the level of the middle of the thalamus. Note the shrinkage of the thalamus on the left side. Note, also, that the whole extent of the cortex on the left side has undergone complete pathological destruction with the exception of the gyrus cinguli, the hippocampal formation, the hippocampal gyrus, and a narrow strip of the inferior temporal convolution.

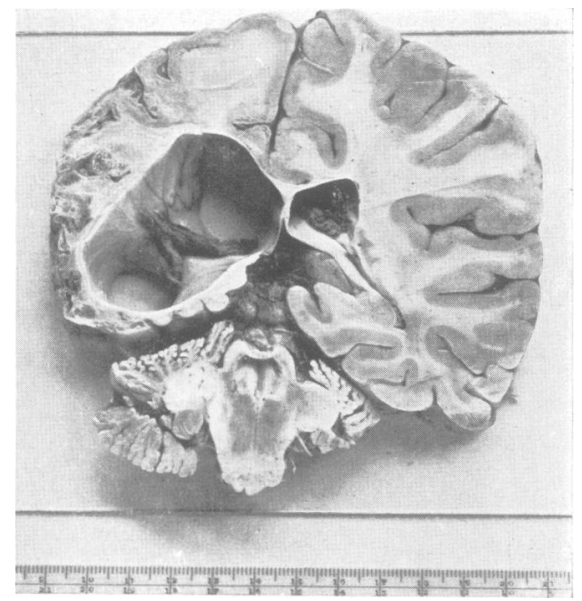

Fig. 3.-Section of the brain behind the level of the thalamus. Note that the upper end of the central convolutions remains partly intact (in macroscopical appearance), and that the visual cortex has been stretched out to form a thin layer in the medial wall of the dilated posterior horn of the lateral ventricle. The choroid plexus is adherent to its lateral wall. 
The left lateral ventricle was greatly dilated (Fig. 3). Its ependyma was everywhere smooth and glistening except where a tag of the left choroid plexus had become adherent to the lateral wall in the temporo-occipital region (Fig. 3). This choroid plexus appeared in other respects normal. The corpus callosum was greatly atrophied, measuring from $0.25 \mathrm{~cm}$. to $0.3 \mathrm{~cm}$. in thickness. There was also great atrophy of the left optic thalamus, details of which are separately described below. The left internal capsule and crus were considerably wasted.

Spinal Cord.-There was no obvious alteration in structure; the anterior spinal roots and horns appeared equal and normal.

\section{Microscopic Examination}

Methods.-Blocks were selected from representative parts of the central nervous system both for paraffin and for frozen sections. Paraffin sections were stained with Ehrlich's hæmatoxylin and eosin, Weigert's iron hæmatoxylin and van Gieson's mixture, Weigert's fuchselin and neutral red, phosphotungstic-acid hæmatoxylin, Roussy and Lhermitte's modification of Nissl's method and the Loyez hæmatoxylin method for myelin. Sections cut on the freezing microtome were stained with Scharlach R. for fatty substances, Spielmeyer's method for myelin, Bielschowsky's and Rio-Hortega's methods for neurofibrils and Penfield's modification for oligodendroglia and microglia.

Left Cerebral Hemisphere.-The leptomeninges over the left cerebrum are thickened wherever convolutional atrophy is present. This thickening appears to be due to redundancy of the meninges since there is no obvious increase of fibrous tissue, and the texture is spongy. In places there is sparse infiltration of the deep surface of the arachnoid membrane and of the pia immediately over the cortex by small lymphocytes, occasional neutrophil leucocytes, and mononuclear cells. The blood-vessels everywhere appear normal with the exception of a single arteriole in the frontal region in which slight focal hypertrophy of the intima has produced a crescentic thickening. The adjacent media is normal.

The cortex is almost everywhere greatly atrophied, being replaced by a spongy tissue in which anastomosing trabeculæ of densely felted neuroglial fibrils enclose spaces of varying size and shape (Fig. 4). These spaces sometimes contain a few foam cells and, more rarely, small lymphocytes. In the most severely affected areas all the neurones have disappeared; occasionally they persist as "fossils" encrusted with iron salts (Fig. 5). In the less severely affected parts, for example the cingulate gyrus and the visual cortex, the neurones are well preserved. Transitional stages can be distinguished in passing from these areas towards those that have undergone atrophy ; in these the neurones show progressively greater degrees of distortion of shape, eccentricity of the nucleus, and margination and chromatolysis of Nissl substance. Accompanying these changes is a progressive gliosis, shown by the presence in silver preparations of astroblastic and binucleated forms, and stages in the development of porous areas (Fig. 6). In the intact and less affected areas the microglial cells are either normal or show slight amœboid swelling and occasional rod forms. The oligodendroglial cells likewise are normal or slightly swollen. In the more 


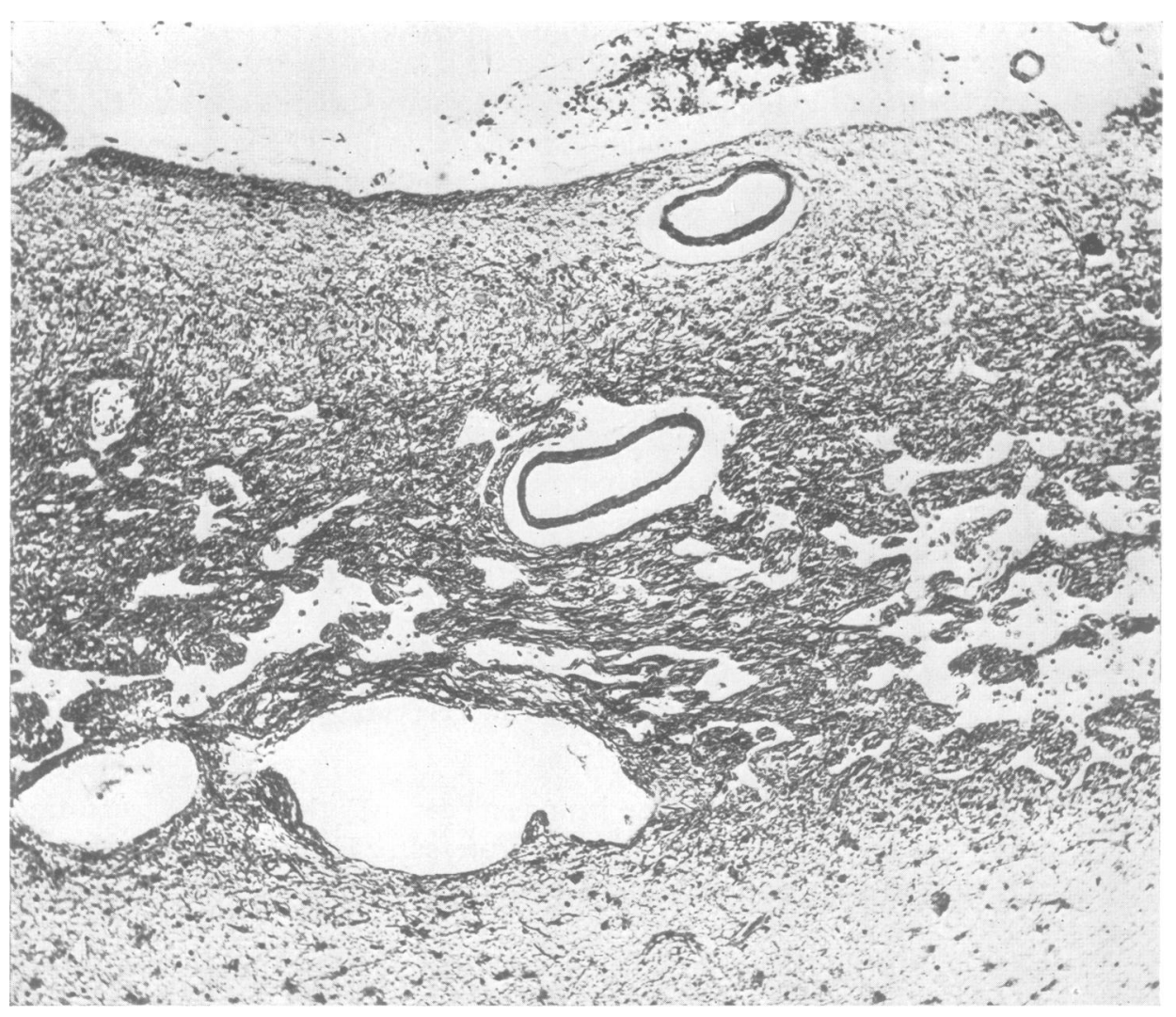

Fig. 4.-Left occipital cortex showing replacement of cortex by dense glial feltwork enclosing irregular spaces. The deep border of the cortex is represented by the larger oval space to left of centre. Phosphotungstic acid hæmatoxylin. $\times 93$.

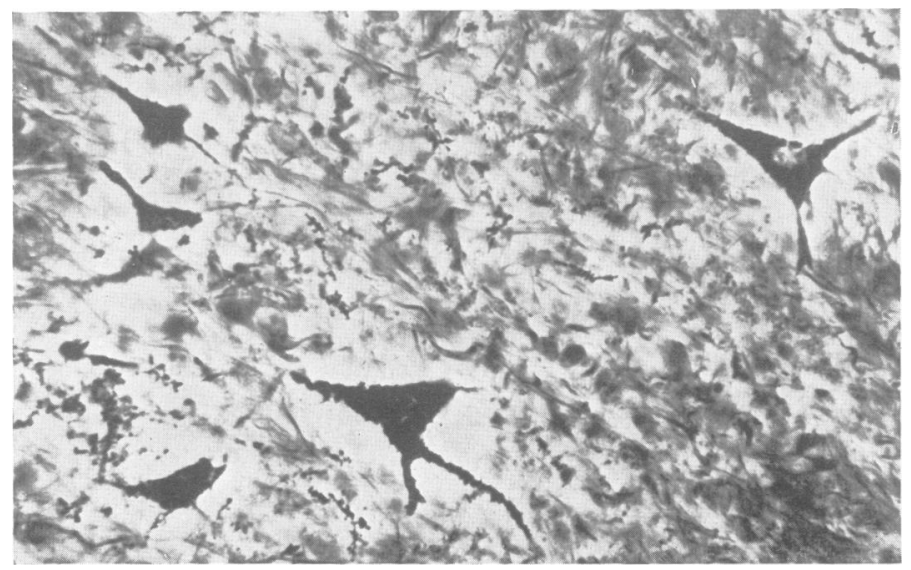

Fig. 5.-Left motor cortex showing pyramidal cells encrusted with iron salts. Amongst them are many similarly encrusted fragments of dendrites. Rio-Hortega's silver carbonate for neurofibrils. $\times 490$. 
severely affected parts the microglial cells are scarce; amœboid forms are occasionally present in the walls of the cavities, and compound granular corpuscles containing globules of fat are often present singly or in small groups within the cavities. The oligodendroglial cells are scanty ; in strong contrast to those in the more normal areas and in the right cerebrum they are invariably devoid of processes, greatly atrophied, and contain shrunken pyknotic nuclei.

Silver preparations to demonstrate connective tissue show a dense network

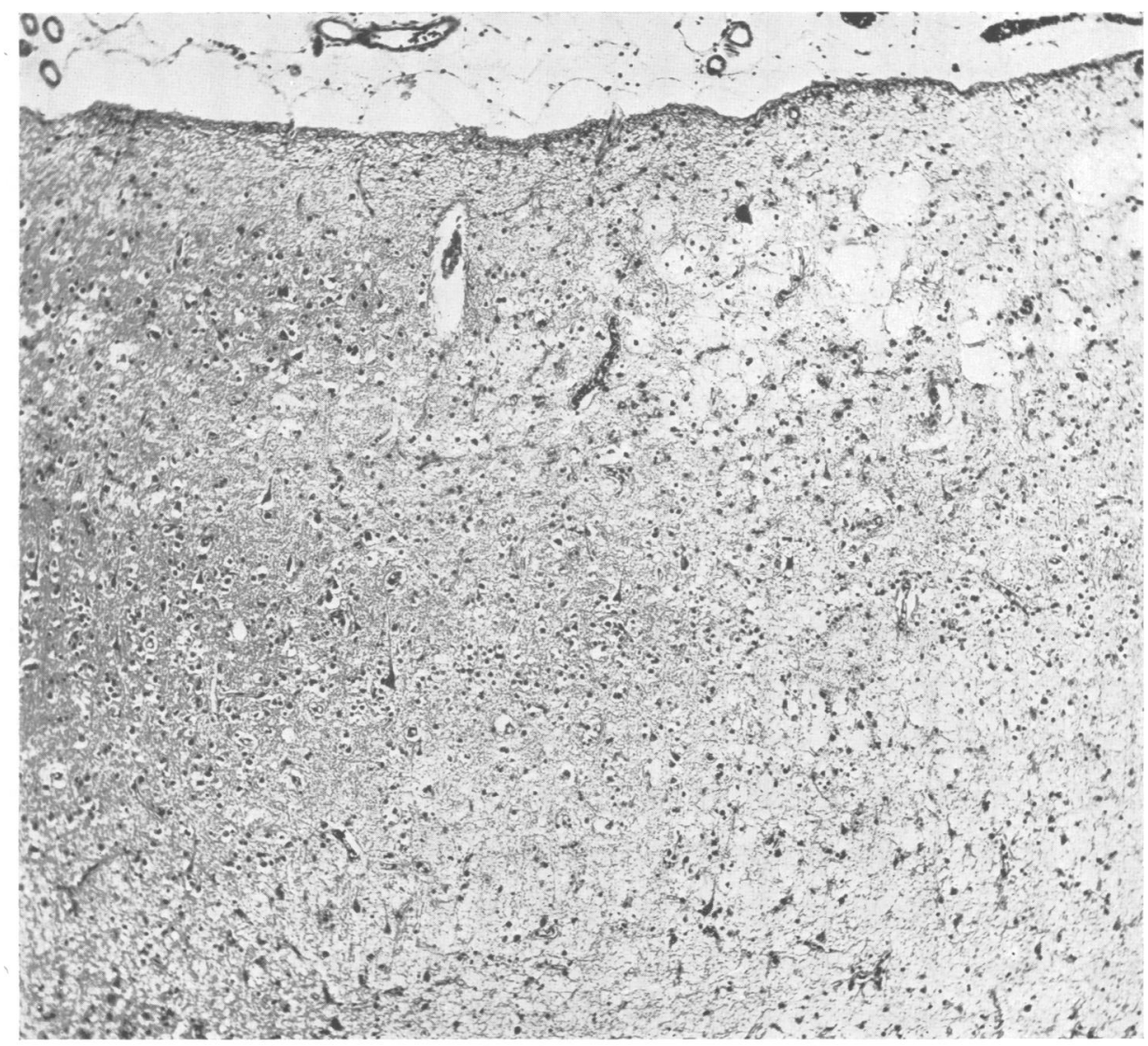

Fig. 6.-Cortex near area striata showing, on right, early stages in formation of cavities with disappearance of neurones. Phosphotungstic acid hæmatoxylin. $\times 76$.

of blood-vessels with slightly thickened walls throughout the atrophied parts of the cortex. There is, however, no histological evidence of any new formation of vessels : the appearances suggest that their prominence is due to condensation secondary to atrophy.

In the white matter there is an almost complete loss of myelin beneath affected parts of the cortex accompanied by a dense and relatively acellular gliosis (Figs. 7, 8). The few surviving myelin sheaths frequently show ballooning and fragmentation. Beneath unaffected parts of the cortex, such as the 
area striata, the white matter appears normal. The transition between the normal and abnormal parts is gradual and it is remarkable that, in the transi-

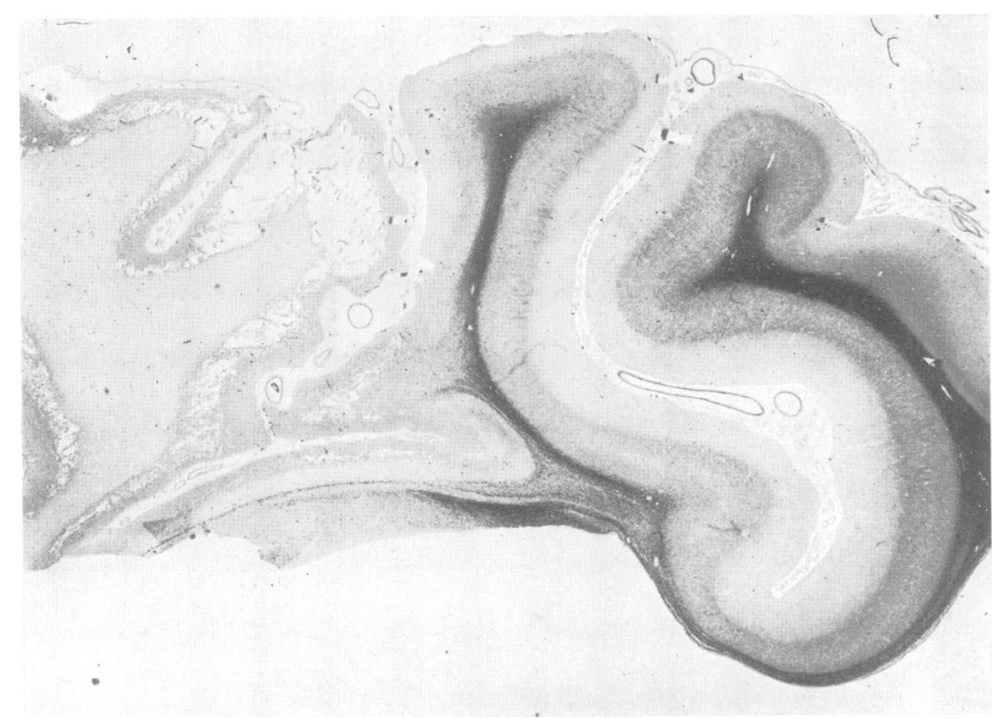

Fig. 7.-Left occipital cortex showing preservation of area striata on right. Cortical destruction and complete demyelination of neighbouring gyrus. Loyez' hæmatoxylin. $\times 4 \cdot 2$.

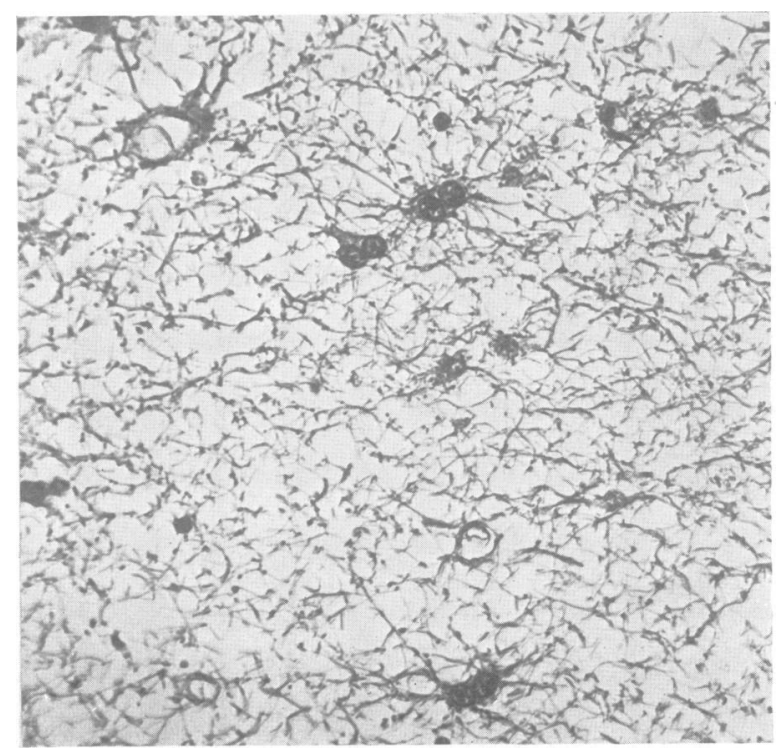

Fig. 8.-Subcortical tissue from left occipital lobe showing replacement of white matter by astrocytes. Two binucleated cells are present near centre of field. Phosphotungstic acid hæmatoxylin. $\times 380$.

tional areas examined, the changes in the white matter appear rather more advanced than those in the overlying cortex. 
The character of the gliosis differs from that in the cortex both in texture and in the form of the cells. In the cortex densely felted bundles of piloid astrocytes are present in all severely affected areas ; in the white matter the interlacing neuroglial fibres are less dense and the cells are stellate. Frequently they are binucleate (Fig. 8). Compound granular corpuscles containing fatty substances are distributed rather sparsely and irregularly in the affected white matter ; they sometimes form cuffs in the Virchow-Robin spaces of the bloodvessels. The scarcity of oligodendroglial cells is more remarkable here than in the cortex ; all show advanced atrophy as already described.

Sections through the site of attachment of the choroid plexus to the lateral wall of the ventricle in the parieto-occipital region show fibrous union between the stroma of the plexus and the ependyma. There is no inflammatory reaction in the adjacent tissues. The choroid plexus appears normal apart from the presence of a few calcospherites in its stroma.

Representative sections from different parts of the right cerebral hemisphere show no histological abnormality apart from the occasional presence of small lymphocytes and sparser neutrophil leucocytes in the leptomeninges.

A similar sparse cellular infiltration is present in the leptomeninges over the cerebellum. The left cerebellar hemisphere appears normal. All folia of the right cerebellar hemisphere are slightly but unequally atrophied. The atrophy is accompanied by a partial, sometimes marked, loss of both Purkinje cells and granule cells and by a gliosis due to proliferation of the Bergmann cells. There is no demonstrable change in the white matter.

The spinal cord is normal apart from a descending pyramidal degeneration on the right side.

\section{Changes in the Left Optic Thalamus}

Before describing the distribution of the cell atrophy in the thalamus, it is desirable to refer again to the precise extent of the cortical destruction. As already noted, almost all the cortex has been completely disorganized by the pathological process, and even deep-lying tracts of cortex, such as the whole of the insular area, have been involved. Certain regions, however, have escaped destruction. The visual striate cortex, it is interesting to note, has been left intact so far as its intrinsic structure is concerned (Fig. 7). Sections of this area, stained with toluidin blue, show the various cell-laminæ to be quite well defined, each lamina containing its characteristic cells in an apparently normal condition. It is not possible to affirm that no cell atrophy at all has occurred in the visual cortex, but, if it has occurred, it is too slight to be detected by any change in cytoarchitectural appearance.

A strip of the cingulate gyrus which is in immediate relation to the corpus callosum has also escaped complete destruction (Fig. 2). Sections have shown, however, that the cytoarchitecture of this cortex is not normal, for, while numbers of well-formed pyramidal cells are still present, there is evidently a general atrophy affecting all the cell layers. The immediately subjacent white matter, also, shows a marked gliosis. 
On the convex surface of the brain, the whole extent of the cortex has been destroyed with the exception of a small portion of the upper end of the central convolutions (Fig. 3). Here, as in the cingulate gyrus, there are marked atrophic changes. As in the case of the striate cortex, the distribution of the incompletely involved cortex in the gyrus cinguli and the central convolutions suggests a relation to blood-supply, for these areas of the cortex are vascularized by the main branches of the anterior cerebral artery.

The only other portions of the cortex to escape involvement are the dentate gyrus, the hippocampus, the hippocampal gyrus, and a narrow strip of the inferior temporal convolution. Like the striate area, these cortical structures are supplied by the posterior cerebral artery.

In summary, it may be noted that all that part of the cortex comprising the vascular territory of the left middle cerebral artery has undergone complete disorganization. The same process has also extended in some degree into the territories of supply of the anterior and posterior cerebral arteries except along the distribution of the main cortical branches of these vessels.

Macroscopically, the left thalamus is seen in sections to be considerably shrunken in size (Fig. 2). Spaced serial sections of the whole of the thalamus of both sides were prepared, and stained with toluidin blue. These were systematically studied in order to determine the extent of the cellular atrophy which had occurred. The results of this study may most conveniently be described by reference to individual nuclear elements.

Anterior Nucleus-This element (which corresponds to the nucleus anteroventralis of lower mammals) has undergone complete degeneration with replacement of the cells by a dense gliosis, except in the rostral half of the nucleus where there is a small circumscribed cluster of apparently normal cells in its medio-ventral part. In the caudal half of the nucleus no normal cells could be found. It has now been well established that the nucleus antero-ventralis projects on to the gyrus cinguli (Le Gros Clark and Boggon, 1932), and it undergoes complete atrophy following experimental hemidecortication in the chimpanzee (Walker, 1938). The persistence of a few cells in the present case is almost certainly related to the fact that a strip of the cingulate gyrus has escaped complete destruction.

Ventral and Lateral Nuclei-These elements of the thalamus have undergone practically complete degeneration. In rostral planes, occasional isolated and normal cells are seen at the ventro-lateral margin of the ventral nucleus, but it is difficult to determine whether these belong to the ventral nucleus proper, or to that part of the nucleus reticularis which is in immediate relation to it. In the caudal part of the thalamus, a slender strand of scattered cells extends dorso-laterally through the region of the ventral nucleus (Figs. 9, 10). This line of cells becomes even less distinct more posteriorly, and is finally reduced to odd scattered cells in the same region which are not easy to find except by careful scrutiny of the sections. Apart from these exceptions, and the presence of a very occasional cell to be found by searching over wide fields, all the neurones of the lateral and ventral nuclei have disappeared and are replaced by an exceptionally dense gliosis. It is reasonable to assume that 
the persistence of some of the cells is related to the fact that a small portion of the central gyri has escaped complete destruction by the pathological process.

Following hemidecortication in the chimpanzee and monkey, Walker $(1935,1938)$ found the rostral extremity of the ventral nuclear mass free from degeneration, suggesting that in these animals this element (nucleus ventralis anterior) does not project on to the cerebral cortex. In the decorticated cat, Waller (1938) found the persistence of normal cells in the medial part of the anterior ventral nucleus, while, in similar experiments, Bard and Rioch (1937)

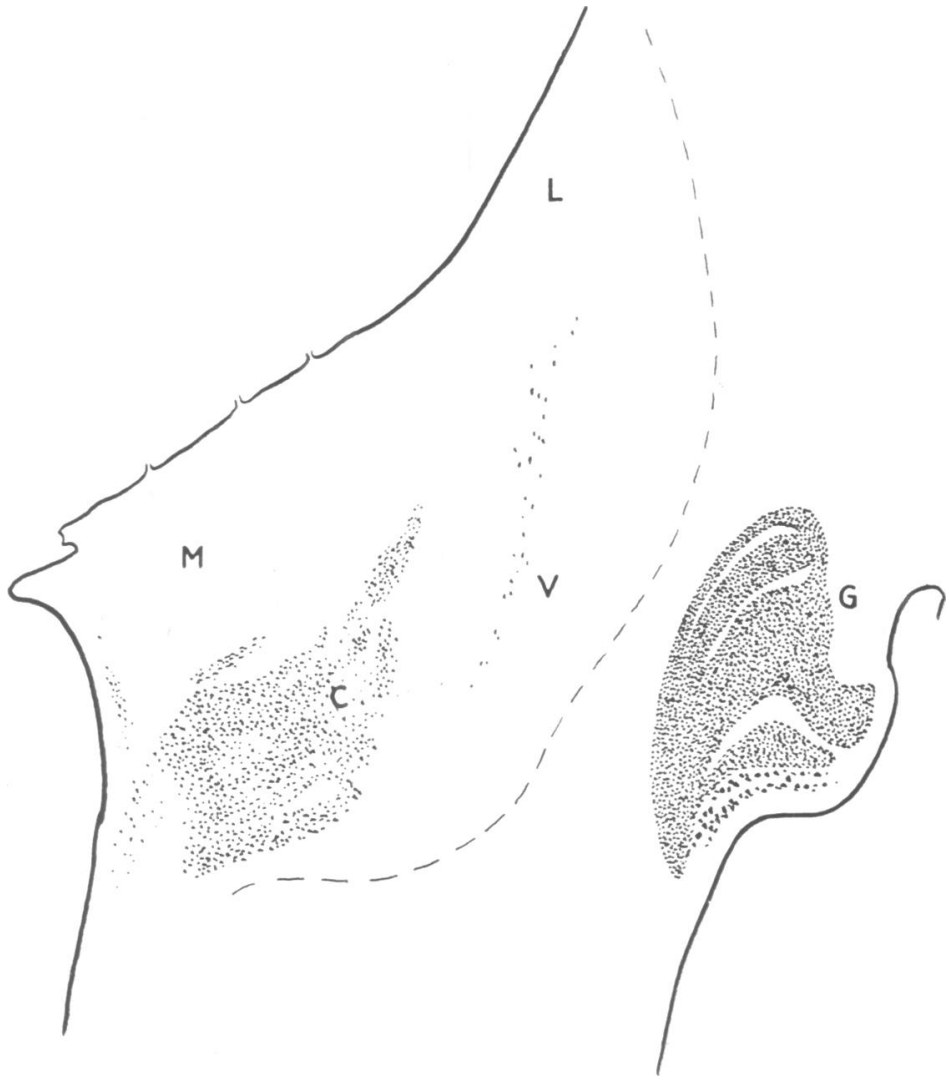

Fig. 9.-Drawing of a section through the thalamus at the level of the anterior part of the centre median nucleus (traced with a projection apparatus). C. Centre median nucleus. G. Lateral geniculate body. L. Lateral nucleus. M. Medial nucleus. V. Ventral nucleus. $\times 5$.

found the nucleus almost completely degenerated, with only occasional normal cells persisting. In the present case, the cells occupying the topographical position of the nucleus ventralis anterior have apparently undergone total degeneration, and it may be inferred, therefore, that in the human thalamus the whole antero-posterior extent of the ventral nuclear mass is entirely concerned with the projection of ascending fibres to the cortex.

Medial Nucleus (Nucleus Dorso-medialis of Lower Mammals.)-This element shows complete degeneration, except only for the appearance in odd sections of a very occasional isolated cell, or a cluster of cells (up to seven or 
eight), which can be found by careful searching. It is now established that the medial nucleus projects on to the prefrontal cortical fields. While in lower mammals this is certainly true for most of the cells of the medial nucleus except for some larger neurones at its medial margin, the present case suggests that in the human brain it applies to the entire nucleus.

Pulvinar.-No normal nerve cells could be found in any part of the pulvinar -everywhere the normal cytoarchitecture is replaced by a dense gliosis.

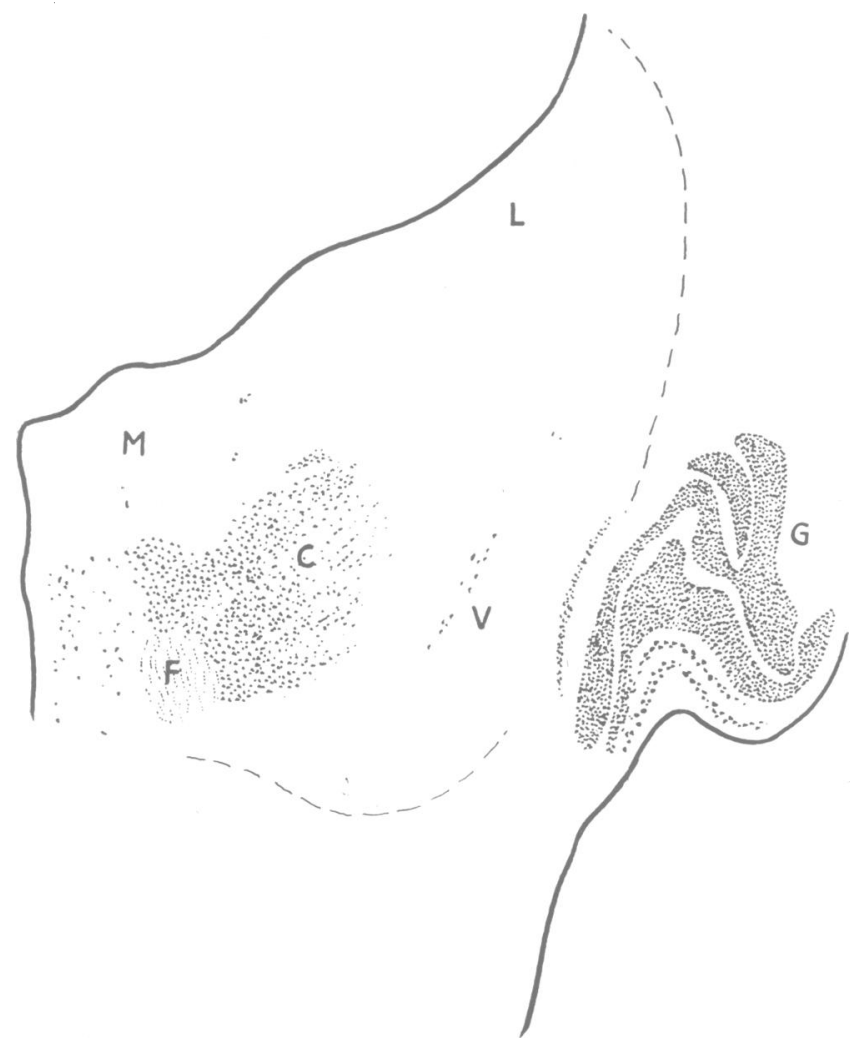

Fig. 10.-Drawing of a section through the thalamus at the level of the posterior part of the centre median nucleus (traced with a projection apparatus). F. Fasciculus retroflexus. Other lettering as in previous figure. $\times 5$.

Experiments involving isolated cortical lesions have shown that the pulvinar projects mainly on to the posterior parietal cortex (Le Gros Clark and Northfield, 1937), and the element has been shown by Walker to undergo complete degeneration following decortication in the monkey and chimpanzee.

Centre Median Nucleus.-This thalamic element persists apparently unchanged. Surrounded by areas of total cell atrophy and conspicuous gliosis, it stands out in toluidin blue sections quite sharply defined (Figs. 9, 10, 11). Its cells stain normally, and, so far as can be judged by direct examination of the sections and comparison with the opposite thalamus, they show their normal density. The ground-substance of the nucleus, however, shows a slight degree of gliosis which may be related to the degeneration of fibres of 
passage, or of descending cortical connections. It is, of course, not possible to affirm that none of the cells of the centre median nucleus have undergone degeneration ; it is possible to say, however, that certainly the great majority, and probably all, of them remain intact.

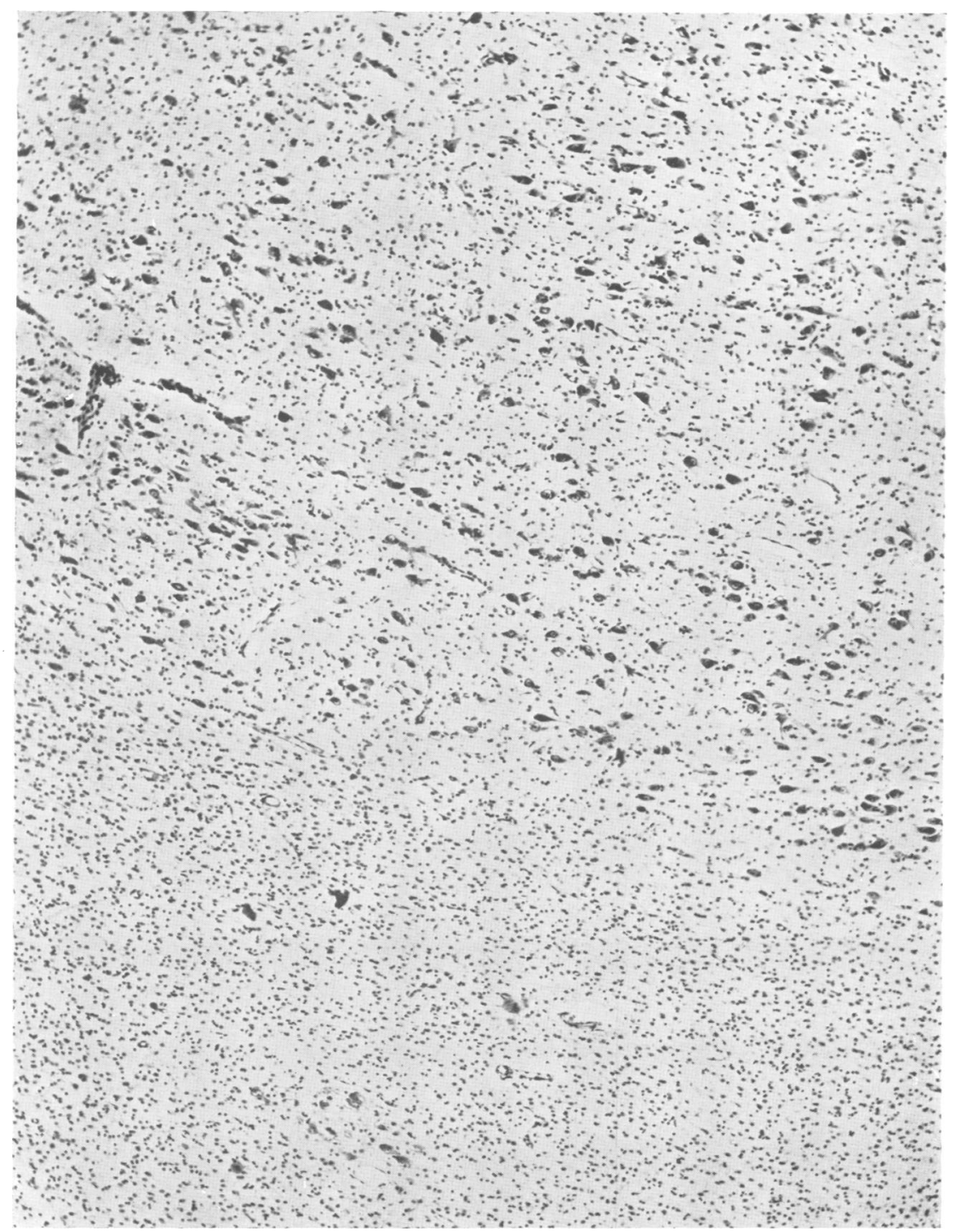

Fig. 11.-Microphotograph of a section showing (above) a part of the centre median nucleus with intact cells, and (below) the region of the ventral nucleus in which the cells have disappeared and have been replaced by a dense gliosis. $\times 55$.

The persistence of the centre median nucleus in the midst of degenerated areas following hemidecortication has been noted by Walker in the chimpanzee and monkey, whence it appears that this element does not send any projection fibres to the cerebral cortex. On the other hand, its connections remain quite 
obscure. In a pathological case reported recently (Le Gros Clark and Ritchie Russell, 1939), in which a vascular lesion had isolated the insular cortex, the centre median nucleus was found to have undergone practically complete degeneration, while most of the adjacent nuclei were unaffected. This observation suggested the possibility that the centre median nucleus may project on to the insular cortex and, in order to test this, the insular area in a macaque monkey was completely destroyed by the method of devascularization. In this experiment, however, the nucleus remained intact, and it is quite apparent from this and from the previous experiments of other authors that in the monkey it is not connected with the insula. On the other hand, the possibility still remained that it might do so in the human brain in which the insula shows the development of new cytoarchitectural areas. This possibility is now ruled out by the present case.

The suggestion that the centre median nucleus sends its efferent fibres to the corpus striatum has been entertained by a number of authorities. More recently, some experimental evidence has been adduced in support of this view. Walker (1938a) found complete degeneration of the nucleus in a monkey after decortication with marked damage to the basal ganglia, and, since in other experiments in which the caudate nucleus and putamen only had been partly destroyed the element remained normal, he suggested that it probably projects on to the globus pallidus portion of the basal ganglia. In the pathological case to which reference has just been made, however, the globus pallidus was intact and the vascular lesion had only involved a relatively small portion of the putamen of the lentiform nucleus. Since this limited striatal lesion was judged to be hardly adequate to account for the practically complete atrophy of the centre median nucleus, the efferent connections of the latter still remain in doubt.

Dr. J. G. Greenfield has recently permitted one of us to examine sections of the thalamus from a case of Kernicterus lately described (FitzGerald, Greenfield, and Kounine, 1939). In this case (No. 1) the ganglion cells of the globus pallidus "had almost completely disappeared, only sparse shrunken cells remaining." Nevertheless, the sections show that the centre median nucleus appears quite normal in its nerve-cells and its glial content.

Lateral Geniculate Body.-The cells of all the laminæ of this visual centre appear to be completely normal. This is somewhat surprising considering the degree to which the cortex of the occipital lobe has been stretched out to a thin layer in the wall of the dilated lateral ventricle. On the other hand, the fact that the lateral geniculate body remains normal in structure, while all parts of the pulvinar have undergone complete degeneration, is in harmony with the observation that normal striate cortex is rather sharply circumscribed by completely degenerated cortex, for it is known from experimental work that some elements of the pulvinar project on to the peristriate area which immediately surrounds the visual cortex (Le Gros Clark and Northfield, 1939).

Medial Geniculate Body.-This nucleus has undergone complete degeneration as the result of the destruction of the audito-sensory cortex.

Nuclei of the Mid-line.-Throughout the thalamus on the affected side, 
a thin zone of small nerve-cells persists in a subependymal position along the wall of the third ventricle. These cells are surprisingly few in number as compared with the cells of the mid-line nuclei which are reported to remain intact in lower mammals after decortication.

Intralaminar Nuclei.-In lower mammals, the main thalamic nuclei are outlined by strands of cells occupying the position of medullary laminæ, and these intralaminar nuclei persist after decortication. Some of them are well enough defined anatomically to merit description as separate nuclear elements of the thalamus. In the human brain they are less conspicuous, and in the present case they are not evident at all. There is a complete absence of cells corresponding in position to the nucleus paracentralis and the nucleus centralis lateralis of lower mammals, for instance. From the rostral end of the centre median nucleus a strand of cells extends for a short distance dorso-laterally into the lower part of the medial medullary lamina (Fig. 9), but except for this extension there is no persistence of the intralaminar nuclei.

Nucleus Reticularis.-This thin layer of cells has apparently undergone complete degeneration except possibly in rostral planes where (as already noted) some normal cells at the lateral margin of the ventral nucleus are to be found intact.

\section{Comment}

In this case an acquired hemiplegia in childhood is correlated pathologically with a conspicuous degree of atrophy and porencephaly of the left cerebral cortex and with advanced demyelination and gliosis of the white matter of that hemisphere. Although infantile hemiplegia has usually been ascribed to a congenital defect or to birth trauma, it is generally recognized that post-natal examples also occur. Most of these are due to circulatory disturbances such as sinus thrombosis, or embolism secondary to infective endocarditis. Such disturbances are ruled out in the present case both by the observations at necropsy and the morbid anatomy of the brain. An infective origin in certain of these cases is postulated by Wohlwill (1936) who suggests that, when the onset is acute and accompanied by high fever, the condition should be regarded as a "genuine encephalitis." The clinical features of this type are described by Taylor (1905) as follows : " A child hitherto healthy and without hereditary stigmata becomes ill without any apparent cause, between the ages of one month and six years. The early symptoms are severe, and consist of fever, convulsions, often vomiting and coma. These symptoms persist from twenty-four hours to a week ; and towards the end of the period, as the general condition improves, hemiplegia is noticed to come on, first in the face, then in the arm, and lastly in the leg. The hemiplegia becomes severe and the limbs remain flaccid for some days, after which the flaccidity begins to disappear and the limbs become spastic. In some cases the paralysis disappears completely. In most cases a greater or less degree of paralysis or perversity of movement remains." The pathology of this type of acquired hemiplegia is, however, still obscure. Strümpell $(1885)$ claimed that it represented the cerebral form of infantile poliomyelitis, but the existence of such a condition is now disputed. Pette 
(1936) declares that, in a wide experience, he has never seen a case and considers it wiser to assume that it does not exist. Grinker (1937) considers that this so-called Strümpell-Leichtenstern type should be regarded as an acute syndrome, the ætiological factors of which may be varied.

While infantile poliomyelitis cannot be absolutely ruled out in the present case, the absence of any histological changes in the anterior horn cells is against this diagnosis. The clinical history and results of examination of the cerebrospinal fluid point strongly, however, to an initial acute inflammation of the central nervous system. The relatively small number of cells in the lumbar spinal fluid suggests that the initial condition was an encephalitis rather than a meningitis. The ætiological agent, however, has not been demonstrated. The histological examination, while disclosing an unusual kind of cerebral destruction, yielded no information bearing on this point. The changes in the white matter of the left hemisphere bore a close resemblance to those in Schilder's encephalitis, but the condition as a whole differs strongly from this disease in the conspicuous involvement of the cortex, the unilateral involvement of the brain and absence of any evidence of progression from the occipital to the frontal lobe. In the involvement of the cortex and the development of porous areas there is perhaps a stronger resemblance to certain examples of diffuse progressive cerebral sclerosis in infants (Russell and Tallerman, 1937).

Amongst cases reported in the literature the nearest approach to the present one was found in the two examples described by Bielschowsky (1917-18). In his first case there was an acute onset at the age of five years, accompanied by fever, convulsions, and a skin rash, and followed by hemiplegia. Epileptic convulsions persisted during the following eighteen years and the patient died, an imbecile, at the age of 23 . In his second case the initial attack, characterized by severe convulsions and loss of consciousness, occurred at 6 years ; it was followed by weakness of the right side and loss of speech. Later there was an improvement in speech but the hemiplegia persisted. Epileptiform convulsions and deterioration of intelligence became pronounced and the patient died in postepileptic coma at the age of 14 . In both these cases the brain showed a unilateral atrophy similar to that described in our present case except that cortical destruction was less severe and the sub-cortical white matter showed neither demyelination nor gliosis. The lateral ventricle on the atrophied side was grossly dilated as in the present case. This dilatation obviously differs from a true internal hydrocephalus in that no obstruction is present : it is the result of atrophy with retraction of the tissues. A bilateral dilatation is similarly found in examples of progressive cerebral sclerosis of infants. Bielschowsky's cases again resembled ours in the preservation of the area striata and lateral geniculate bodies, and in the well-marked atrophy of the optic thalamus.

With respect to the thalamic atrophy in our case it may be said, in general, that the only elements which persist undegenerated are the lateral geniculate body, the centre median nucleus, and a narrow subependymal zone of small cells. ${ }^{*}$ The lateral geniculate body in the primates is known to project entirely

* The elements of the epithalamus (habenular and paratænial nuclei) are not considered here. These elements are not affected by the degeneration which involves the thalamus proper. 
on to the visual cortex, and in the present case this cortical area had escaped pathological destruction. The occasional normal cells which are still to be found in the anterior, ventral, and other nuclear elements (to which reference has already been made) almost certainly owe their survival to incomplete destruction of the cortical areas to which they are related. The conclusion naturally follows that every nuclear element of the human thalamus is entirely concerned with the projection of impulses on to some part of the cerebral cortex, with the exception only of the centre median nucleus and the subependymal zone of cells. This conclusion is based on the assumption that the atrophy of the cells is the direct result of the interruption by the cortical destruction of their axonal processes. The possibility that the atrophy may be related to vascular disturbances or that it may be a transneuronal atrophy consequent on the interruption of descending cortico-thalamic fibres must also be considered. In regard to the first, it may be said that there is no evidence in the sections of any interference with the vascular supply of the thalamus, and the persistence of sharply circumscribed normal elements in the midst of degenerated areas is inconsistent with such an interpretation. As regards the possibility of transneuronal atrophy, we may note that in this type of atrophy, such as may occur in the lateral geniculate body after section of the optic nerve, the cells undergo considerable shrinkage and chromatolytic degeneration, but the cell-bodies do not show the complete disappearance which typically occurs in most neurones of the central nervous system after section of their axonal processes. In any case marked transneuronal atrophy is an unusual phenomenon in the brain and, so far as is known, is limited here to the lateral geniculate body. Experiments by one of us has shown that it does not occur in the sensory nuclei of the thalamus after interruption of ascending sensory tracts, and even in the lateral geniculate body it does not occur to any conspicuous extent in some mammals (e.g. rat and ferret). Lastly, the type of degeneration and accompanying gliosis observed in the present case is precisely similar to the reaction which is seen in different thalamic elements after localized ablation of the cortical areas on to which they are known to project.

It appears, then, that in the human thalamus, the centre median nucleus is the only element which does not project on to the cortex (apart from the few small cells of the subependymal zone). This observation clearly has an important bearing on the question as to how far thalamic activity can proceed independently of the cerebral cortex in man. Unfortunately nothing certain is known regarding the connections of the centre median nucleus. We have noted the possibility that it may project on to the corpus striatum, but we have also pointed out that the case of Le Gros Clark and Ritchie Russell, in which complete degeneration of the centre median nucleus was associated with a very limited lesion of the putamen, does not lend much support to this view. Several authors have described the termination in the centre median nucleus of ascending sensory tracts, particularly fibres of the trigeminal fillet. Here again, the evidence is equivocal, for it can be demonstrated that at least some of these fibres merely pass through the nucleus on their way to rostral levels of the ventral nucleus (Le Gros Clark, 1936). The centre median nucleus has 
sometimes been regarded as a phylogenetically ancient element of the thalamus, mainly it seems because of its medial position. Comparative anatomical studies, however, are not in harmony with this conception, for, in the primates at least, the nucleus is much smaller and less clearly defined in lower forms, and becomes progressively better differentiated and relatively larger in an ascending scale of primates leading up to Man. However, the possibility must be entertained that the nucleus, in the circumscribed form in which it is found in higher primates, may represent intralaminar nuclear elements which have been displaced and collected together in one mass as the result of pressure exerted by the growth of the main nuclei of the thalamus in among which they lie in lower mammals.

In conclusion, it may be noted that striatal connections have been ascribed to a number of thalamic nuclei (besides the centre median nucleus) by various authors. The fact that in the present case these nuclei have undergone complete atrophy, while the basal ganglia have not been affected by pathological destruction, is not consonant with this view. In other words, it has yet to be proved that any of the nuclear elements of the thalamus proper are directly connected by efferent fibres with any part of the corpus striatum.

\section{Summary}

Clinical and pathological details are given of a case in which a hemiplegia, acquired in childhood, is associated with diffuse atrophy of the corresponding cerebral hemisphere and conspicuous atrophy of the optic thalamus.

With the exception of a few specified areas the cortex is replaced by spongy glial tissue from which almost all the neurones have disappeared. The result is practically equivalent to an experimental hemidecortication.

Thalamic atrophy is practically complete on the affected side with the exception of the centre median nucleus, the lateral geniculate body, and a narrow subependymal zone of small cells. From this it is concluded that all other parts of the thalamus are concerned with the projection of impulses on to some part of the cerebral cortex. The connections of the centre median nucleus remain obscure.

\section{REFERENCES}

Bard, P., and Rioch, D. M. (1937). Johns Hopk. Hosp. Bull., 60, 73.

Bielschowsky, M. (1917-18). J. Psychol. Neurol. Leipzig, 22, 225.

FitzGerald, G. M., Greenfield, J. G., and Kounine, B. (1939). Brain, 62, 292.

Grinker, R. R. (1937). Neurology. Baillière, Tindall and Cox, London.

Le Gros Clark, W. E. (1936). J. Anat., 71, 7.

and Boggon, R. H. (1932). Ibid., 67, 215.

- and Northfield, D. W. C. (1937). Brain, 60, 126.

and Russell, W. Ritchie (1939). J. Anat., 73, 255.

Pette, H. (1936). Bumke's Handb. Neurol., 13, Part 2, 208.

Russell, D. S., and Tallerman, K. H. (1937). Arch. Dis. Child., 12, 71.

Strümpell, A. (1885). Jb. Kinderheilk., 22, 173.

Taylor, J. (1905). Paralysis and other diseases of the nervous system in childhood and early life. J. and A. Churchill. London.

Walker, A. E. (1935). J. comp. Neurol., 62, 407.

- (1938a). The Primate Thalamus. Univ. Chicago Press.

- (1938b). J. comp. Neurol., 69, 487.

Waller, W. H. (1938). J. Anat., 72, 475.

Wohlwill, F. (1936). Bumke's Handb. Neurol., 16, 35. 